\title{
Developmental Risk: Evidence from Large Nonright-Handed Samples
}

\author{
Filippos Vlachos, ${ }^{1}$ Francois Gaillard, ${ }^{2}$ Kiriazis Vaitsis, ${ }^{1}$ and Argiris Karapetsas ${ }^{1}$ \\ ${ }^{1}$ Department of Special Education, University of Thessaly, Argonafton \& Filellinon, 38221 Volos, Greece \\ ${ }^{2}$ Institute of Psychology, University of Lausanne, Géopolis, 1015 Lausanne, Switzerland \\ Correspondence should be addressed to Filippos Vlachos; fvlachos@uth.gr
}

Received 29 March 2013; Revised 4 June 2013; Accepted 19 June 2013

Academic Editor: Ross Flom

Copyright (C) 2013 Filippos Vlachos et al. This is an open access article distributed under the Creative Commons Attribution License, which permits unrestricted use, distribution, and reproduction in any medium, provided the original work is properly cited.

\begin{abstract}
The aim of the present study is twofold. First, we tested the view that individuals who do not develop a typically strong behavioral laterality are distributed differentially among the two genders across age. Second, we examined whether left handedness and mixed handedness are associated with an elevated risk of some developmental or cognitive deficits. A special recruitment procedure provided norms of the Rey-Osterrieth Complex Figure (ROCF) copy from large samples of left-handed $(N=420)$ and mixedhanded $(N=72)$ compared to right-handed $(N=420)$ schoolchildren and adults $(N=545)$. This graphic task was considered as reflective of the growth of visual-spatial skills and impairment at copying as a developmental risk. Subjects' hand preference was assessed by the Edinburgh Handedness Inventory. Data analysis indicated that (1) the trend towards consistent right handedness is sex related. Girls are clearly ahead of boys in this lateralization process, and boys are overrepresented in mixed-handed subjects. The greater prevalence of mixed-handed boys compared to girls decreases with age. (2) Performance on drawing the ROCF varies according to age and handedness groups. Mixed-handed subjects scored worse in all age groups. The results are discussed in relation to the hormonal-developmental, neuropathological, and learning theories of lateralization.
\end{abstract}

\section{Introduction}

Handedness is a significant feature of ontogenetic development. Its consistency and stability provide evidence for brain hemispheric specialization and can be used as an indicator of developmental stages. Lateralization is both cause and consequence of having a brain with two cerebral hemispheres specialized to perform different tasks and work together in order to improve many motor and cognitive tasks. At the first glance, no hand lateralization is observed in young children who have not yet learned to use innate biological asymmetry. However, careful observation of motor behavior in infants already reveals the human specific trend towards dextrality $[1,2]$.

The study of handedness has been of interest for many years because subtle cognitive and behavioral differences have been demonstrated in relation to various handedness measures [3]. Gender differences in handedness are widely reported. A recent meta-analysis of 144 studies [4] demonstrated that the gender difference in handedness is both significant and robust, indicating that the overall best estimate, albeit not universal, for the male to female odds ratio was 1.23 . The purpose of this study is to investigate further the effects of handedness, as a proxy for hemispheric laterality, in terms of gender differences, performance on a cognitive task, and the potential for later cognitive impairment, examining large left-handed and mixed-handed groups of children and adults. Large samples of nonright handers are rare because only $9-10 \%$ of the population of schoolchildren is left-handed, and there are even fewer nonlateralized children. In previous studies [5-7], using a special recruitment procedure in order to obtain a large group of left handers (each lefthanded child was matched by age and sex to a right-handed child), we have demonstrated differences between left and right handers on the Rey-Osterrieth Complex Figure (ROCF) test, a popular tool used to test visuospatial-constructional functions [8]. In the present study, we complete this observation by including non-lateralized children. We are also adding 
a group of adults in order to present the whole cognitive development.

A very recent study [9] indicated that there is no single pattern in the development of handedness from 6 to 24 months, and the shift to more robust hand use preferences may be a developmental phenomenon. Inconsistent and unstable handedness has been found in several clinical groups with pathological cognitive development $[10,11]$. However, many studies showed no relationship between handedness and cognitive development in samples of normal children [12-16]. These observations show that factors that disrupt cognitive development can also disrupt the development of consistent manual dominance. We can explain this discrepancy between apparently contradictory results by considering progressive handedness to be merely a sign of brain specialization and not a condition of mental development.

However, during the school years, consistent and stable handedness appears as a tool for learning fine motor tasks such as writing and for developing the spatial sense necessary for written language and calculation. Although nonlateralized schoolchildren are not delayed in maturation, they lack the advantage that left and right handers have in their learning. This is why nonlateralized schoolchildren are often at risk of being considered as slow learners or as learning disabled as far as visual-spatial abilities are concerned. In this sense, they are subjected to a true developmental risk.

Progressive hand preference indicates neurophysiological asymmetry which is genetic and biological in origin [1720]. However, some handedness conditions are pathological. According to the theoretical account of pathological lefthandedness syndrome, a subgroup of left handers suffers from a condition that involves an early injury. This syndrome is believed to be caused by a hemispheric lesion that is predominantly left-sided (or bilateral asymmetric), which onsets before the age of 6 and which encroaches upon the critical speech zones of the frontotemporal/frontoparietal cortex [10]. Indeed, the trend towards functional lateralization reveals itself to be sensitive to any cerebral disturbance. The neuropathological hypothesis of handedness would firstly predict that diffused brain injury would result in a lack of hand dominance and, secondly, that lateralized hemispheric damage would produce strong ipsilateral hand dominance.

In the first case, patients present a habitus of mixed handedness characterized by low motor performances on both body sides. Research on prematurity, on risk pregnancy, and on dystocia has shown that very often these conditions are associated with cognitive deficits and an increased frequency of inconsistent right handedness. These studies suggest that the brain might be affected as a whole resulting in mixed handedness and bilateral clumsiness [21-24]. Additionally, several studies consider mixed handedness as a pathological condition [13, 25-27].

In the second case, some of the $100 \%$ left handers are suffering from right hand handicap corresponding to left brain damage $[1,10,28]$. The non dominant hand of such strongly lateralized persons actually acts as a symptomatic limb. Obstetricians and pediatricians estimate that $1.5-6 \%$ of children are likely to suffer from brain damage early in life [29, 30]. Thus, it is reasonable to expect to have some of these brain damaged patients in a sample of 912 children. Surprisingly there seems to be no literature on pathological $100 \%$ right handedness, which is, of course, the reverse possibility. However, the neuropathological hypothesis only accounts for one explanation of the variation in the relationship between handedness and skills. Table 1 presents five hypotheses related to this variation.

The genetic influence on laterality has been proved by comparing parental with child handedness both in normal and adoptive families [31-33]. At present, laterality genetics is far from clear. Some claim that there may be a single gene influencing laterality development with homozygotically and heterozygotically different conditions [17]. Others believe that the inheritance process is polygenetic [19], and the recent molecular studies strongly favor a polygenic model. More specifically, a few years ago Francks et al. [40] identified an imprinted gene, LRRTM1, within the 2p12-q11 region, which is expressed during the development of specific forebrain structures. The paternal copy of this gene was associated with both left handedness and schizophrenia, and this was the first potential genetic influence on human handedness identified, and the first putative genetic effect on variability in human brain asymmetry. Other researchers [41] identified the X-linked androgen receptor as a candidate gene for handedness. In another study of genetic influences on handedness [42] researchers after analyzing a large sample of twin and family data for hand preference concluded that the familial aggregation for hand preference was found to be consistent with additive genetic effects, which accounted for about a quarter of the variation in the trait with the remainder accounted for by nonshared environmental influences. All these findings are consistent with the emerging view that handedness is determined by multiple interacting genetic and environmental factors. A recent study [43] showed that human handedness is affected by early lateralised visual experience, thus leading the researchers to the suggestion that a combined gene-environment model could better explain the development of human handedness.

Hormones also influence brain development, as has been demonstrated by comparing female and male brain lateralization and specialization $[44,45]$. Female brains show weaker lateralization, but this sex difference only accounts for 1 or $2 \%$ of the variance in laterality [46]. Clinically, women suffer less than men from aphasia following brain damage. They also seem to recover better than males when similar aphasic states are observed [47]. There is a slight preponderance of nonright-handed males in child clinical samples where girls appear as more lateralized than boys [34, 48-50]. According to Geschwind and Galaburda's hypothesis [34] there is a fundamental link between abnormal left-hemisphere development, reduced dextrality, various learning disorders, and various immune related and other medical conditions, which is forged by the prenatal influence of testosterone on the developing nervous system. The authors propose that excessive levels of fetal testosterone influence the rate of maturation of the cerebral hemispheres which, in turn, may increase the probability of nonright handedness and learning disorders, affecting males more than females due to the higher rates of testosterone [34]. Two decades ago, a review by 
TABLE 1: Influence on the lateralization process: evidence and implication.

\begin{tabular}{|c|c|c|}
\hline Type of influence & Effect evidenced & Hypothesis for research \\
\hline Genetic & $\begin{array}{l}\text { Children with left-handed parents more } \\
\text { often become left handers but not in } \\
\text { adopted samples. }\end{array}$ & $\begin{array}{l}\text { Becoming left-handed is as physiological as becoming } \\
\text { right-handed. } \\
\text { Some people do not inherit the genetic influence, therefore } \\
\text { lateralizing themselves by the other influences or even by } \\
\text { chance. } \\
\text { Healthy genetic left-handed develop an original mind in } \\
\text { comparison with right-handed individuals [31-33]. }\end{array}$ \\
\hline Hormonal/developmental & $\begin{array}{l}\text { Greater prevalence of nonright } \\
\text { handedness in males. Cerebral } \\
\text { lateralization more pronounced in males. }\end{array}$ & $\begin{array}{l}\text { Male's overall maturational gap. } \\
\text { Male’s overall boost of right-hemispheric functions [34]. }\end{array}$ \\
\hline Neuropathological & $\begin{array}{l}\text { Any brain injury before, during and after } \\
\text { birth contradicts the lateralization } \\
\text { process. }\end{array}$ & $\begin{array}{l}\text { Results depend on global or focal brain suffering. If global, } \\
\text { some ambidextrous subjects suffer bilateral brain damage. If } \\
\text { focal, some } 100 \% \text { right-handed and some } 100 \% \text { left-handed } \\
\text { suffer hemispheric dysfunction }[1,10,28] \text {. }\end{array}$ \\
\hline Neuropsychological & $\begin{array}{l}\text { Each hand shows an ability advantage } \\
\text { corresponding to the specialization of the } \\
\text { direct linked hemisphere. }\end{array}$ & $\begin{array}{l}\text { The right hand is better at communicating, and the left hand } \\
\text { is better at visual-spatial tasks [35-37]. }\end{array}$ \\
\hline Learning & $\begin{array}{l}\text { Children take progressively advantage of } \\
\text { their lateralization by building } \\
\text { body-space relationships and developing } \\
\text { constructional abilities. }\end{array}$ & $\begin{array}{l}\text { Lateralized children develop a better spatial sense than } \\
\text { nonlateralized ones. } \\
\text { Risks for academic learning are lower in lateralized than } \\
\text { ambidextrous children. } \\
\text { Target: arithmetic, eidetic reading, and writing }[38,39] \text {. }\end{array}$ \\
\hline
\end{tabular}

Bryden et al. [51] questioned the Geschwind and Galaburda's hypothesis [34] as a cohesive entity. However, this same review did acknowledge an empirical basis for the association of a number of developmental and health problems with hand preference and hormone action. Even though a strong version of the link between handedness with hormone action has been refuted [51], still numerous reports exist to point to some role of hormone action in hand preference. For example, a few years ago [52] pointed out that the length of the index finger relative to that of the ring finger (the $2 \mathrm{D}: 4 \mathrm{D}$ ratio) is a marker of the amount of testosterone that was present in the foetal environment. Recent studies $[53,54]$ confirmed that the difference between the digit ratios of the right and left hands was a significant predictor of handedness.

There are two more hypotheses that predict a relation between lateralization and neuropsychological development: neuropsychology and learning. Concerning neuropsychology, brain hemispheric specialization directly influences hand specialization. Despite the fact that every human being acts in a bihemispheric manner, the right hand is more effectively used for left-hemispheric functions and the left for right-hemispheric functions. This does not mean that the right hand cannot obey the right hemisphere, but experimental studies have shown that the right hand is simply better than the left hand in performing left hemisphere specialized tasks [35-37]. For our purpose this means that we could predict that the left hand is better than the right in copying the ROCF. Of course, we did not ask our right handers to draw with their left hand in order to check this prediction. However, in a recent study aiming to investigate intermanual differences in copying performance, Yamashita [55] examined the right- and left-hand performance on the ROCF in a nonclinical sample of undergraduates. Yamashita found that hand use had a minimal effect on performance in the copy trial. So the hemispheric specialization theory does not imply that left handers will better succeed in copying the ROCF than right handers. Indeed, a dominant hand for specific tasks is the result of long training which can interfere with hemispheric specialization. This is particularly true for drawing and writing.

As far as learning is concerned, lateralization provides a child with a tool for space behavior, since lateral preference strongly helps the child to build his/her body scheme and organize peri corporal behavior. This boosted training results in a certain advantage in developing visual-spatial abilities, reading, and even calculation in lateralized children $[38,39]$.

The aim of the study reported here is twofold. First, we tested the view that individuals who do not develop a typically strong behavioral laterality are distributed differentially among the two genders across age. Second, we examined whether left handedness and mixed handedness are associated with an elevated risk of some developmental or cognitive deficits. To this end, we used two popular tools, the Edinburgh Handedness Inventory (EHI) [56] and the Rey-Osterrieth Complex Figure (ROCF) test [57-59] in order to assess handedness and cognitive development, respectively. EHI is a reliable and well-validated instrument and, according to the Citation Index, has been the most widely used handedness inventory in the literature. The ROCF is a popular neuropsychological test designed for adults that has been successfully employed with school-aged children. Its utility with this population stems largely from the fact that it provides such a rich data source, allowing for the assessment of multiple cognitive processes (e.g., perceptual, spatial, metacognitive, and memory). The ROCF has proven useful in the study of normal (Karapetsas and Kantas, 1991 [60]; 
Waber and Holmes, 1985 [61]) and atypical child development (Brandys and Rourke, 1991 [62]). Children's performance on the ROCF follows a clear developmental trajectory; it is very challenging for early primary school children but becomes more manageable for older children and adults [60, 61].

The five hypotheses concerning the origin and the consequences of handedness in cognitive development mentioned in Table 1 could explain why experimental results often contradict each other depending on case selection and on the handedness characteristics considered. The present study deals with three of them: hormonal-developmental, neuropathological, and learning. In line with the delayed development of the left hemisphere in males proposed by Geschwind and Galaburda [34], we expect to find a preponderance of boys in the nonlateralized group because of their slight delay in brain maturation when compared to girls (Hypothesis 1). According to the neuropathological model $[1,10,28]$, it was hypothesized that performance on copying the ROCF would be particularly low in mixedhanded and in extremely lateralized persons (Hypothesis 2). Finally, the learning model of handedness [38, 39] would predict that the worst scores on ROCF would be found in mixed-handed subjects, because non-lateralized children lack laterality support in the development of their skills (Hypothesis 3).

\section{Method}

2.1. Population. We followed a two-stage process for our case selection. Children were recruited from normal schools using a procedure which aimed at collecting as many left handers as right handers. Teachers were asked to indicate which students wrote with their left hand. Using the Edinburgh Handedness Inventory [56], we subsequently identified 420 children as left-handed and 72 children as nonlateralized. Then, each lefthanded child was matched by age and sex to a right-handed child. By the end of this procedure, we collected observations from a total sample of 912 schoolchildren consisting of 420 left handers, 420 right handers, and 72 mixed handers. All of these children were drawn from regular schools and had no history of major medical illness, psychiatric illness, developmental disorder, or significant visual or auditory impairments according to the medical reports of their schools. The 912 children were subdivided into two age groups. The younger age group ( 5.5 to $9.5 \mathrm{yr}$.) consisted of 512 subjects, and the older group (aged 9.5 to 12.5 yrs.) consisted of 400 subjects.

The adults were 545 students (199 men and 346 women), aged 17.6 to 32 years $(M=19.5, \mathrm{SD}=1.8)$, all drawn from the Engineering Faculty and the Humanities Faculty of the University of Thessaly, Greece. University students in Greece represent 30 percent of the total young adult population, so we selected a subgroup of the total population at the highest academic level. Given the overrepresentation of women in our adult sample and the study's aim to compare the gender ratio of adults with that of children, we calculated a corrective index (dividing the number of men by that of women), in order to represent men and women equally. The corrective index applied was number of women $\times 0.575=$ corrected number of women compared to men.

\subsection{Materials and Procedure}

2.2.1. Lateralization Index. In the present study we used a shortened version of the EHI [56] to assess hand preference. Following Bryden and Steenhuis' recommendation [63] we saved the following items from the original version: writing, drawing, use of scissors, striking a match, and opening a box. The five items listed are deemed sufficient to produce reliable and valid results [64] and allow for safe classification of the participants into handedness groups [65]. Numerous studies have confirmed the superior rigour and reliability of the EHI compared to other handedness questionnaires [66-69]. Test-Retest reliability of the EHI as measured by the Pearson $r$, Kendall $\tau$, and Spearman $r_{s}$ ranges from 0.95 to 0.98 [68]. The medium to high correlations of the EHI with other behavioral measures of handedness (e.g., the Purdue Pegboard test, a test of manual dexterity) demonstrate the high concurrent validity of the test [70-72].

Procedure. For children aged 5.5 to 7.5 , the assessment of hand preference was done through demonstration in order to avoid any interpretational difficulties that could occur with a written questionnaire. For each handedness questionnaire item, children were asked to indicate whether they had used the right hand or the left hand. This type of procedure has been proposed by Coren [65] as the most appropriate means for assessing handedness in younger groups. Further, Steenhuis and Bryden [73] indicated that preference questions are closely related to performance measures concerning similar activities.

Scoring. Children aged 7.5 to 12.5 , and adults completed the EHI. The score for the EHI is computed as the difference between the total number of right- or left-handed responses divided by the total number of responses, and the result is multiplied by 100 to produce the Laterality Quotient. Subjects who scored between +91 and +100 were labelled consistent right-handers $(\mathrm{CRH})$, those with scores between +30 and +90 inconsistent right-handers (IRH), those with scores between -29 and +29 mixed-handers $(\mathrm{MH})$, those with scores between -30 and -90 inconsistent left-handers (ILH), and those with scores between -91 and -100 consistent left-handers (CLH).

2.2.2. Visual-Spatial Skills. In order to examine visual-spatial skills, we used the Rey-Osterrieth Complex Figure (ROCF), according to the directions provided by the authors [57-59].

Procedure. The figure was displayed on a piece of white paper measuring $30 \mathrm{~cm} \times 21 \mathrm{~cm}$ so that the base rectangle measured $8.0 \times 5.5 \mathrm{~cm}$. When tested, each participant was provided with a piece of white paper of the same dimensions as the one on which the Rey-Osterrieth figure was presented. Participants were tested in their classroom groups. After the figure had been administered, we asked participants 
to copy it as best they could without neglecting any of its finer details. There were no time restrictions. When a participant stopped, we asked him/her if he/she had finished. If he/she answered affirmatively, we removed the copying sheet. All the left handers used their left hand for the drawing task, and all the right handers used their right hand. Mixed-handed people were allowed to draw with either hand.

Scoring. To evaluate the data in the manner described by Osterrieth [59], the complex figure was divided into 18 elements, each of which was assigned the same value. Then the number of elements in each figure reproduced by the participants was counted, their positions in relation to the whole figure were noted, and the accuracy of reproduction was noted. The following scoring method was employed. For each element that was correctly placed in its proper position and, moreover, was accurately drawn, a score of 2 was allocated. If the element was correct, but, in the wrong position, a score of 1 was allocated. If the element was distorted or incomplete but recognizable and in the right position a score of 1 was allocated. If the element possessed the same characteristics and was misplaced, a score of 0.5 was allocated. Finally, if an element was not easily recognisable or was absent, the score was zero. The highest possible score for each figure was 36 points. The time required for the completion of the figure was not taken into consideration during scoring, but on average copying took between 3 and 5 minutes. A random sample of 80 figures was scored by two scorers on the basis of the aforementioned criteria, with $94 \%$ agreement. This indicated sufficient interrater reliability of the scoring method, and thus the remaining figures were scored by one judge only.

\section{Results}

3.1. Sex Ratio in Lateralized and Nonlateralized Samples. We found unequal numbers of boys and girls among the mixedhanded children: 27 boys and 7 girls in the younger group (a ratio of $3.6: 1$ ) and 27 boys and 13 girls in the older group (a ratio of $2.1: 1$ ). Taking into account the corrected number of women, in order to be able to make comparisons with the data of children, we found a sex ratio of 1.4 men for 1 woman in mixed-handed adults. A Kruskall-Wallis test indicated a significant relationship between sex ratio and age in mixedhanded people $(H=5.76, d f=2$, and $P=.018)$. Table 2 recapitulates the frequencies and sex ratio according to handedness and age groups.

Table 2 shows that mixed handedness exists in all age groups. However, there are three times more mixed-handed boys than girls in the younger group. Maturation and learning change the figures. However, mixed handedness is still more frequent in adult males than females.

The right-handedness figures confirm the existence of a relationship between sex and handedness development. Indeed, because left-handers were matched to right-handers for both boys and girls, we would expect to find equivalent frequencies of inconsistent and consistent right-handers regardless of sex. This was held true for younger children where we observed 57 IRH boys compared to 59 IRH girls and $63 \mathrm{CRH}$ boys compared to $61 \mathrm{CRH}$ girls $\left(\chi^{2}=.07, d f=\right.$ $1, \mathrm{~ns})$. However, a developmental trend seems to be observed regarding sex ratio in right-handers since we found $41 \mathrm{IRH}$ boys compared to 28 IRH girls and $49 \mathrm{CRH}$ boys compared to $62 \mathrm{CRH}$ girls in older children $\left(\chi^{2}=3.97, d f=1\right.$, and $P=$ .046). To examine whether the older right-handed children were making a transition from the inconsistence use of the right hand to the consistence use and the possible effect of sex a Kruskall-Wallis test was performed. The significance test between younger and older children revealed a main effect for hand consistency $\left(\chi^{2}=4.16, P=.041\right)$ and a main effect for sex ratio $\left(\chi^{2}=18.01, P<.001\right)$. Thus, even when applying a strict recruitment procedure in order to balance left-handed and right-handed boys and girls, we found a tendency for older right-handed girls to become more strongly lateralized than boys with age, and sex-related developmental trends appeared to exist.

To summarize, we observed a sex-related developmental change in handedness distribution. This refers to the hormonal-developmental hypothesis of lateralization. Both the diminishing prevalence of boys in the mixed-handed sample and the stronger lateralization of older right-handed girls compared to boys indicate a relative delay in the lateralization process of boys.

\subsection{Age, Sex, and Handedness Differences in Visual-Spatial} Skills (Intelligence). ROCF raw scores show systematic changes across ages (see Table 3 ). Scores were evaluated using a $5 \times 3 \times 2$ (handedness $\times$ age $\times$ sex) mixed-effect analysis of variance. There were statistically significant main effects for handedness $\left(F_{4,1427}=25.26, P<.001\right)$ and age $\left(F_{2,1427}=182.46, P<.001\right)$, which means, respectively, that performance improved with age (as can be seen in Table 3) and that handedness groups differed in their performance. The main effect between males and females was not statistically significant $\left(F_{1,1427}=3.19\right.$, ns). There was also a statistically significant age by handedness interaction $\left(F_{8,1427}=154.39, P<.001\right)$, which indicates that the effect of handedness differed at different ages. No other significant interaction between the factors was found (handedness $\times$ sex $F_{4,1427}=0.59$, ns; age $\times \operatorname{sex} F_{2,1427}=1.47$, ns; handedness $\times$ age $\times \operatorname{sex} F_{8,1427}=0.63$, ns).

In order to identify the differences in ROCF performance by handedness in the various age groups, we applied oneway analysis of variance followed by Duncan tests for post hoc comparisons between the handedness groups. The results of these analyses revealed statistically significant differences between handedness groups in the younger children $\left(F_{4,507}=\right.$ 14.99, $P<.001)$. Duncan tests showed that there were significant differences between the mixed-handed group $(\mathrm{MH})$ and all the other groups. The difference in ROCF mean scores regarding handedness was also statistically significant in older children $\left(F_{4,395}=20.23, P<.001\right)$. The Duncan test showed that the $\mathrm{MH}$ group differed significantly from all the other groups. As was previously demonstrated, left handers did not perform as well as right handers $[5,6]$. This was true for children in both age groups. In adults, the difference 
TABLE 2: Sex ratio (males/females) according to handedness and age groups.

\begin{tabular}{|c|c|c|c|c|}
\hline Handedness groups & Lateralization index & Younger children & Older children & Adults \\
\hline $\mathrm{CRH}$ & +100 & 1.0 & 0.8 & 0.9 \\
\hline IRH & +90 to +30 & 0.9 & 1.5 & 1.1 \\
\hline $\mathrm{MH}$ & +20 to -20 & 3.6 & 2.1 & 1.4 \\
\hline ILH & -30 to -90 & 1.0 & 0.9 & 0.8 \\
\hline $\mathrm{CLH}$ & -100 & 1.0 & 1.1 & 1.0 \\
\hline
\end{tabular}

Note: CRH: consistent right-handers, IRH: inconsistent right-handers, MH: mixed handers, ILH: inconsistent left handers, CLH: consistent left-handers.

TABLE 3: Rey-Osterrieth Complex Figure scores by handedness and age group.

\begin{tabular}{lcccccccc}
\hline \multirow{2}{*}{ Handedness group } & \multicolumn{2}{r}{ Younger children } & \multicolumn{2}{c}{ Older children } & \multicolumn{2}{c}{ Adults } & \multicolumn{2}{c}{ Total } \\
& $M$ & SD & $M$ & SD & $M$ & SD & $M$ & SD \\
\hline CRH & 24.7 & 8.5 & 30.3 & 6.3 & 30.9 & 2.9 & 29.2 & 6.2 \\
IRH & 24.7 & 8.8 & 31.9 & 5.0 & 31.5 & 2.5 & 29.6 & 6.2 \\
MH & 16.1 & 5.1 & 22.9 & 5.6 & 29.9 & 2.9 & 21.9 & 7.1 \\
ILH & 21.6 & 7.2 & 27.7 & 5.1 & 31.0 & 2.1 & 24.9 & 7.0 \\
CLH & 18.8 & 6.7 & 27.4 & 5.4 & 31.0 & 2.2 & 23.2 & 7.6 \\
Total & 22.3 & 8.2 & 28.6 & 6.1 & 31.1 & 2.7 & 27.3 & 7.1 \\
\hline
\end{tabular}

Note: CRH: consistent right handers, IRH: inconsistent right handers, MH: mixed handers, ILH: inconsistent left-handers, CLH: consistent left-handers.

in ROCF scores between handedness groups was marginally significant $\left(F_{5,540}=2.38, P=.05\right)$ with the Duncan test showing that the $\mathrm{MH}$ group differed significantly only with the IRH group, which was the handedness subgroup which revealed the higher performance. These differences are presented in Figure 1. The limited differences between handedness groups in adults could be attributed to a ceiling effect as the maximum score was 36 , with an average score being 31.1 with much less variability than in the children samples (2.7 versus 6.1 and 8.2). A similar ceiling effect for the copy measure of the ROCF has been previously reported in a study of college students [74].

\section{Discussion}

The present study investigated whether individuals who do not develop a typically strong behavioral laterality are distributed differentially among the two genders across age and whether left handedness and mixed handedness are associated with an elevated risk of some developmental or cognitive deficits. Thanks to the vast amount of data collected from nonright-handed samples, the present study revealed developmental and sex-related lateralization effects on a task of visual-spatial skills and evaluated these effects with regard to the hypotheses based on the hormonal-developmental, the neuropathological, and the learning models of handedness.

Firstly, based on the hormonal-developmental hypothesis [34] we predicted sex differences in the distribution of handedness across age groups. Our results confirm that the trend towards consistent right handedness is sex related. Specifically, girls in our sample were more advanced than boys in this lateralization process. Moreover, right-handed girls in the older group of this study had become more strongly lateralized than boys. On the other hand, boys were over represented in the mixed-handed group. This tendency

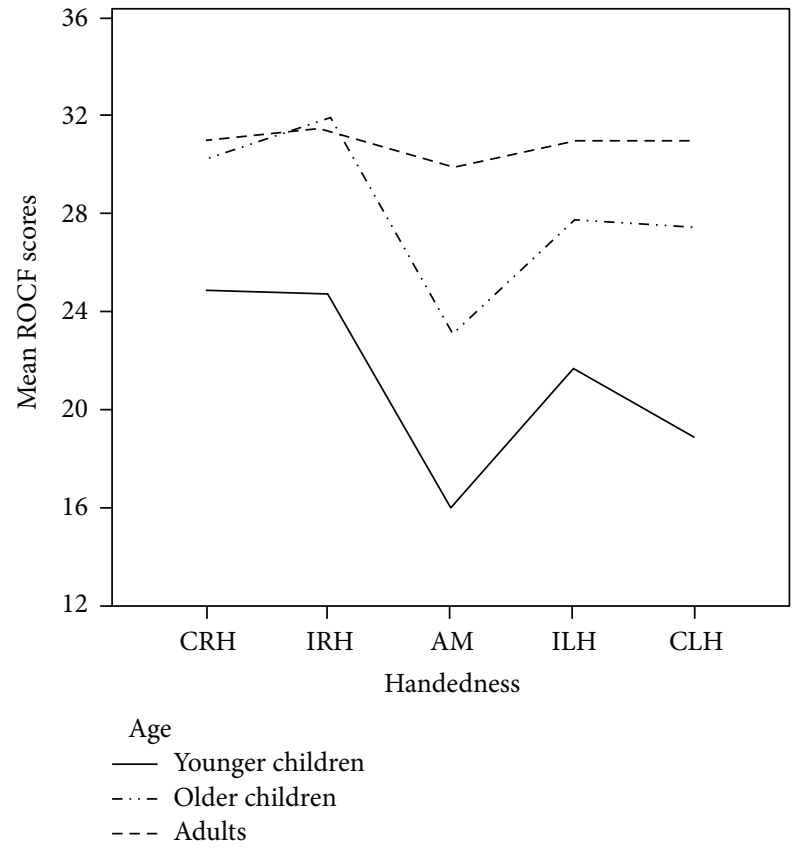

FIgURE 1: Rey-Osterrieth Complex Figure scores by handedness in younger children (-), older children (-.--), and adults (- - -).

appears in our study to continue into adulthood, albeit to a much lesser extent. However, further longitudinal research is needed in order to substantiate this claim. Such longitudinal studies are vital given the discrepancy that exists between neuropsychological studies suggesting that functional brain asymmetry is more characteristic of the right-handed male population and magnetic resonance imaging (MRI) studies [75] pointing to a greater asymmetry in females, at least for motor functions. 
The finding that men are more prone to mixed handedness compared to women is in accordance with that of a recent meta-analysis [4]. Additionally, the same metaanalysis concluded that the magnitude of the difference between sexes was significantly moderated by the way in which handedness was assessed (e.g., by hand writing or by other means). Papadatou-Pastou et al. [4] suggested that, in addition to innate biological differences between the two sexes, handedness is also modulated by psychological influences. This suggestion has been confirmed by the recent study of Dirnberger [76], which examined whether the modulating factors of sex and self-perception (measured as hypochondriac traits) interact and affect handedness in a mixed male and female sample. Their findings suggested that handedness data are influenced by self-perception, attention to action, or inconsistent sensitivity to stimuli from the environment. Nevertheless, the researchers concluded that some modulating psychological or behavioural variables may also underlie genetic influences, which, in turn, might even be sex specific.

The second hypothesis of the present study emanated from the neuropathological model of handedness $[1,10$, 28]. According to this hypothesis the prediction was that performance on copying the ROCF would be particularly low in mixed-handed and in extremely lateralized persons. Our results showed that performance on this visualspatial and graphic task was comparatively low in extremely lateralized persons, who may include symptomatic hemiparesis. The present study complements previous findings $[5,6]$ concerning the left handers' disadvantage in ROCF copying: extreme left handers performed at a lower level in copying than inconsistent left-handers in the younger children's group. Some pathological cases among left-handers might be responsible for this group trend. It could be postulated that pure left handedness emerged precociously in such cases because of an impaired right hand. We know from developmental neuropsychological studies that when the right hemisphere is required to take over language functions in addition to visual-spatial functions, the latter does not develop completely [77]. This might well be the case happening in some of our extreme left-handed youngsters. It is also interesting to note that in the present study extreme lateralization does not correspond to higher cognitive scores than moderate lateralization. Furthermore, the clear disadvantage of extreme left-handedness gradually dissolves with age and completely vanishes in adults. Thus, either our sampling strategy has prevented us from choosing neuropathological cases in the student population, or the visual-spatial impairment is compensated for or even cured by adulthood.

Our third hypothesis based on the learning model $[38,39]$ predicted that low scores on ROCF test would be found in mixed-handed subjects, because these persons lack laterality support in the development of their skills. Backwardness in visual-spatial skills as detected by low ROCF scores is confirmed in mixed-handed children from first to sixth grade.

Overall, the results of this study confirm the learning model predictions but are not in agreement with all the predictions of the neuropathological model. Although poor performance among strong dextral has been found for many types of cognitive and educational tests (for a review see [78]), in our study no differences were found in visual-spatial skills between consistent and inconsistent right handers in this age group. It could be suggested that the appropriate brain mechanisms for successful ROCF copying are established earlier in both right-handed groups. Moreover, another study [79] examined the motor skills of young (approximately seven years old) children and discovered that left- and mixedhanded children were inferior to right-handed children in motor performance. The present data seem to fit in with Crow et al's findings [25] that the most substantial deficits in ability are close to the point of equal hand skill or hemispheric indecision. Crow and his collaborators [25] claimed that the failure to establish unequivocal hemispheric dominance is indicative of developmental delay. Similar findings are supported by research on giftedness. In children aged 8 to 14 , more nongifted than gifted students are distributed in the left- and mixed-handedness categories [80]. Replication of the Crow et al. study [25] by Mayringer and Wimmer [81] was unsuccessful, probably, in our view, for two reasons: firstly the experimental sample involved was relatively modest and biased since the researchers had deliberately brought reading difficulties to the fore (boys only), and, secondly, because hemispheric indecision was less precisely defined. A more recent study [82] using a multiple regression approach, showed that the Mayringer and Wimmer's [81] reanalysis suffered from statistical problems and that the lower scores close to the point of equal hand skill reported by Crow et al. [25] are part of a general monotonic relationship of laterality to cognitive ability, which is independent of overall hand skill. Additionally, our findings are in accordance with recent studies $[83,84]$ which indicated that mixed handedness compared to right handedness was associated with poorer scholastic performance. Overall from the aforementioned studies [25, 82-84] we could conclude that mixed handedness could aid in the recognition of children who are at risk for academic problems.

In adults, however, all handedness groups performed equally. Only a marginal difference in copying the ReyOsterrieth Complex Figure was detected between mixedhanded and the inconsistent right-handed university students. Does this mean that such cognitive difficulties in children are only incidental and can be ignored? Indeed, we do not know if time will complete the gap in visual-spatial development, particularly in the most exposed group, that is, the non-lateralized children. Ignoring what appears to be a simple gap and not a permanent handicap would mean paying little attention to the learning disability condition. For example, the fact that dyslexic children can eventually succeed in professional careers should not prevent consideration of their suffering during development.

We think that this gap in development is pathological both from the pediatric and the psychological points of view. Yeo et al. [85] have shown that left or nonright handers and extreme right handers are characterized by some degree of developmental instability, that is, minor physiological abnormalities. They propose that the underpinnings of 
developmental instability may illuminate the association of handedness with a variety of developmental disorders.

Our results show that delayed learning affects boys three times more than girls in the early school years. Whether they are boys or girls, schoolchildren exhibiting developmental and learning gap deserve our attention in order to prevent academic failure. The sex ratio in mixed-handed schoolchildren reminds us of the prevalence of learning disability which touches two to three times more males than females. The present findings fit in with the epidemiology of learning disabilities.

In sum, even if minority handedness status is not the direct cause of learning disability, as was believed in Orton's time, for example, it nevertheless provides us with an easy means of detecting developmentally "at risk" children. Neuropathological cases are likely to be found in all handedness groups. Some children with mild neurological syndromes are likely to be present among consistent right handers. However, these pathological cases represent a very small percentage of the consistent right-handed group, a larger proportion of consistent left handers, and a much larger proportion of mixed-handed children.

Moreover, recent structural neuroimaging findings [86, 87] have indicated that mixed handedness is associated with neuroanatomical differences, thus offering additional support to the view that individuals who do not develop a typically strong behavioral laterality differ significantly and are at somewhat higher risk of certain disorders and brain abnormalities from consistently left- and right-handed individuals. More specifically, a recent study [86] showed that mixed handedness was associated with increased hippocampal and amygdalar atrophy in ageing. Another study [87] showed that mixed handedness, but not left handedness per se, was associated with corpus callosum thickness, which is indicative of increased interhemispheric interaction. These findings provide evidence for a somewhat different neurobiological background (of either biological or environmental origin) in mixed handers, which might give rise to a number of risk factors leading to certain developmental disabilities.

In conclusion, nonright handedness should not be considered as a risk for cognitive development per se, but the rarity of this condition makes it more likely to count in pathological cases. This has been proven in the present observation by collecting relatively large samples of nonrighthanded children. Furthermore, this supports studies [88] that have found (perhaps excessively) neurological pathology in all non-right-handed subjects, suggesting that future research might focus not so much on left handedness itself but rather on those who lack consistent handedness and/or cerebral asymmetry.

\section{References}

[1] L. Harris and D. Carlson, "Pathological left-handedness: an analysis of theories and evidence," in Brain Lateralization in Children: Developmental Implications, D. L. Molfese and S. J. Segalowitz, Eds., pp. 289-372, Guiford Press, New York, NY, USA, 1988.
[2] J. Liederman and J. Coryell, "The origin of left hand performance: pathological and non-pathological influences," Neuropsychologia, vol. 20, no. 6, pp. 721-725, 1982.

[3] D. Johnston, M. Nicholls, M. Shah, and M. Shields, "Handedness, health and cognitive development: evidence from children in the national longitudinal survey of youth," Journal of the Royal Statistical Society A, vol. 176, pp. 1-20, 2013.

[4] M. Papadatou-Pastou, M. Martin, M. R. Munafò, and G. V. Jones, "Sex differences in left-handedness: a meta-analysis of 144 studies," Psychological Bulletin, vol. 134, no. 5, pp. 677-699, 2008.

[5] A. B. Karapetsas and F. M. Vlachos, "Sex and handedness in development of visuomotor skills," Perceptual and Motor Skills, vol. 85, no. 1, pp. 131-140, 1997.

[6] F. Vlachos, Neuropsychological assessment of visuomotor skills in right and left-handed child [Ph.D. thesis], University of Thessaly, Thessaly, Greece, 1997.

[7] F. M. Vlachos and A. B. Karapetsas, "Visual-motor organization and memory in the right-handed and the left-handed child: a comparative neuropsychological approach," Child Neuropsychology, vol. 2, no. 3, pp. 204-212, 1996.

[8] M.-S. Shin, S.-Y. Park, S.-R. Park, S.-H. Seol, and J. S. Kwon, "Clinical and empirical applications of the Rey-Osterrieth Complex Figure Test," Nature Protocols, vol. 1, no. 2, pp. 892899, 2006.

[9] E. Nelson, J. Campbell, and G. Michel, "Unimanual to bimanual: tracking the development of handedness from 6 to 24 months," Infant Behavioral Development, vol. 36, no. 2, pp. 181-188, 2013.

[10] P. Satz, D. L. Orsini, E. Saslow, and R. Henry, "The pathological left-handedness syndrome," Brain and Cognition, vol. 4, no. 1, pp. 27-46, 1985.

[11] H. V. Soper, P. Satz, D. L. Orsini, W. G. Van Gorp, and M. F. Green, "Handedness distribution in a residential population with severe or profound mental retardation," American Journal of Mental Deficiency, vol. 92, no. 1, pp. 94-102, 1987.

[12] F. Gaillard and P. Satz, "Handedness and reading disability: a developmental study," Archives of Clinical Neuropsychology, vol. 4, no. 1, pp. 63-69, 1989.

[13] D. Bishop, Handedness and Developmental Disorder, Blackwell Scientific Publications, Oxford, UK, 1990.

[14] L. Harris, "Left-handedness," in Handbook of Neuropsychology, I. Rapin and S. J. Segalowitz, Eds., vol. 6 of Child Neuropsychology, pp. 145-208, Elsevier Science Publishers, Amsterdam, The Netherlands, 1992.

[15] D. Natsopoulos, G. Kiosseoglou, and A. Xeromeritou, "Handedness and spatial ability in children: further support for Geschwind's hypothesis of "pathology of superiority" and for Annett's theory of intelligence," Genetic, Social, and General Psychology Monographs, vol. 118, pp. 103-126, 1992.

[16] D. Natsopoulos and A. Xeromeritou, "Verbal abilities of left-and right-handed children," The Journal of Psychology, vol. 123, pp. 121-132, 1989.

[17] M. Annett, Left, Right, Hand and Brain: The Right Shift Theory, Erlbaum, Hillsdale, NJ, USA, 1985.

[18] A. Gessell and L. Ames, "The development of handedness," Journal of Genetic Psychology, vol. 70, pp. 155-176, 1947.

[19] I. McManus, Handedness, Language Dominance and Aphasia: A Genetic Model, Psychological Medicine, Monograph Supplement \#8, Cambridge University Press, 1985.

[20] D. S. Ramsay, "Beginnings of bimanual handedness and speech in infants," Infant Behavior and Development, vol. 3, no. 1, pp. $67-77,1980$. 
[21] M. J. O’Callaghan, D. I. Tudehope, A. E. Dugdale, H. Mohay, Y. Burns, and F. Cook, "Handedness in children with birthweights below 1000 gr," The Lancet, vol. 1, no. 8542, p. 1155, 1987.

[22] G. Ross, E. Lipper, and P. A. Auld, "Hand preference, prematurity and developmental outcome at school age," Neuropsychologia, vol. 30, no. 5, pp. 483-494, 1992.

[23] S. Saigal, P. Rosenbaum, P. Szatmari, and L. Hoult, "Nonright handedness among ELBW and term children at eight years in relation to cognitive function and school performance," Developmental Medicine and Child Neurology, vol. 34, no. 5, pp. 425-433, 1992.

[24] A. Searleman, C. Porac, and S. Coren, "Relationship between birth order, birth stress, and lateral preferences: a critical review," Psychological Bulletin, vol. 105, no. 3, pp. 397-408, 1989.

[25] T. J. Crow, L. R. Crow, D. J. Done, and S. Leask, "Relative hand skill predicts academic ability: global deficits at the point of hemispheric indecision," Neuropsychologia, vol. 36, no. 12, pp. 1275-1282, 1998.

[26] M. Kinsbourne, "Sinistrality, brain organization and cognitive defects," in Brain Lateralization in Children: Developmental Implications, D. Molfese and S. Segalowitz, Eds., Guilford, New York, NY, USA, 1988.

[27] H. V. Soper and P. Satz, "Pathological left-handedness and ambiguous handedness: a new explanatory model," Neuropsychologia, vol. 22, no. 4, pp. 511-515, 1984.

[28] P. Satz, "Pathological left-handedness: an explanatory model," Cortex, vol. 8, pp. 121-135, 1972.

[29] M. L. Levene, J. Kornberg, and T. H. Williams, “The incidence and severity of post-asphyxial encephalopathy in full-term infants," Early Human Development, vol. 11, no. 1, pp. 21-26, 1985.

[30] J. Volpe, Neurology of the Newborn, W.B. Saunders, Philadelphia, $\mathrm{Pa}, \mathrm{USA}, 3$ rd edition, 1995.

[31] M. Annett, "Handedness in families," Annals of Human Genetics, vol. 37, no. 1, pp. 93-105, 1973.

[32] L. Carter-Saltzman, "Biological and sociocultural effects on handedness: comparison between biological and adoptive families," Science, vol. 209, pp. 1263-1265, 1980.

[33] J. Levy, "A review of evidence for a genetic component in the determination of handedness," Behavior Genetics, vol. 6, no. 4, pp. 429-453, 1976.

[34] N. Geschwind and A. Galaburda, Cerebral lateralization, MIT Press, Cambridge, UK, 1987.

[35] J. Levy and C. Trevarthen, "Metacontrol of hemispheric function in human split-brain patients," Journal of Experimental Psychology: Human Perception and Performance, vol. 2, no. 3, pp. 299-312, 1976.

[36] J. Levy, C. Trevarthen, and R. W. Sperry, "Reception of bilateral chimeric figures following hemispheric deconnexion," Brain, vol. 95, no. 1, pp. 61-78, 1972.

[37] R. Sperry, "Lateral specialization in the surgically separated hemispheres," in The Neurosciences: Third Study Program, E. O. Schmitt and F. G. Worden, Eds., pp. 5-19, MIT Press, Cambridge, Mass, USA, 3rd edition, 1974.

[38] B. De Jong, F. Van der Graaf, and A. Paans, "Brain activation related to the representations of external space and body scheme in visuomotor control," NeuroImage, vol. 14, no. 5, pp. 1128-1135, 2001.

[39] O. Simon, J.-F. Mangin, L. Cohen, D. Le Bihan, and S. Dehaene, "Topographical layout of hand, eye, calculation, and languagerelated areas in the human parietal lobe," Neuron, vol. 33, no. 3 , pp. 475-487, 2002.
[40] C. Francks, S. Maegawa, J. Laurén et al., "LRRTM1 on chromosome $2 \mathrm{p} 12$ is a maternally suppressed gene that is associated paternally with handedness and schizophrenia," Molecular Psychiatry, vol. 12, no. 12, pp. 1129-1139, 2007.

[41] S. E. Medland, D. L. Duffy, A. B. Spurdle et al., "Opposite effects of androgen receptor CAG repeat length on increased risk of left-handedness in males and females," Behavior Genetics, vol. 35, no. 6, pp. 735-744, 2005.

[42] S. E. Medland, D. L. Duffy, M. J. Wright et al., "Genetic influences on handedness: data from 25,732 Australian and Dutch twin families," Neuropsychologia, vol. 47, no. 2, pp. 330337, 2009.

[43] S. Ocklenburg, C. Bürger, C. Westermann, D. Schneider, H. Biedermann, and O. Güntürkün, "Visual experience affects handedness," Behavioral Brain Research, vol. 207, pp. 447-451, 2010.

[44] M. Hiscock, R. Inch, J. Hawryluk, P. J. Lyon, and N. Perachio, "Is there a sex difference in human laterality? III. An exhaustive survey of tactile laterality studies from six neuropsychology journals," Journal of Clinical and Experimental Neuropsychology, vol. 21, no. 1, pp. 17-28, 1999.

[45] D. Kimura, Sex and Cognition, MIT Press, Cambridge, UK, 1999.

[46] M. Hiscock, M. Israelian, R. Inch, C. Jacek, and C. HiscockKalil, "Is there a sex difference in human laterality? II. An exhaustive survey of visual laterality studies from six neuropsychology journals," Journal of Clinical and Experimental Neuropsychology, vol. 17, no. 4, pp. 590-610, 1995.

[47] S. F. Cappa and L. A. Vignolo, "Sex differences in the site of brain lesions underlying global aphasia," Aphasiology, vol. 2, no. 3-4, pp. 259-264, 1988.

[48] J. Hellige, Hemispheric Asymmetry: What's Right and What's Left, Harvard University Press, Cambridge, UK, 1993.

[49] J. Obrzut and M. Atkinson, "Relations among learning disorders, handedness, and immune disease," Journal of Clinical and Experimental Neuropsychology, vol. 15, p. 86, 1993.

[50] F. E. Tønnessen, A. Løkken, T. Høien, and I. Lundberg, "Dyslexia, left-handedness, and immune disorders," Archives of Neurology, vol. 50, no. 4, pp. 411-416, 1993.

[51] M. P. Bryden, I. C. McManus, and M. B. Bulman-Fleming, "Evaluating the empirical support for the geschwind-behangalaburda model of cerebral lateralization," Brain and Cognition, vol. 26, no. 2, pp. 103-167, 1994.

[52] J. T. Manning, D. Scutt, J. Wilson, and D. I. Lewis-Jones, “The ratio of 2 nd to 4 th digit length: a predictor of sperm numbers and concentrations of testosterone, luteinizing hormone and oestrogen," Human Reproduction, vol. 13, no. 11, pp. 3000-3004, 1998.

[53] J. T. Manning and M. Peters, "Digit ratio (2D : 4D) and hand preference for writing in the BBC Internet Study," Laterality, vol. 14, no. 5, pp. 528-540, 2009.

[54] A. A. Beaton, N. Rudling, C. Kissling, R. Taurines, and J. Thome, "Digit ratio (2D:4D), salivary testosterone, and handedness," Laterality, vol. 16, no. 2, pp. 136-155, 2011.

[55] H. Yamashita, "Right- and left-hand performance on the reyosterrieth complex figure: a preliminary study in non-clinical sample of right handed people," Archives of Clinical Neuropsychology, vol. 25, no. 4, pp. 314-317, 2010.

[56] R. C. Oldfield, "The assessment and analysis of handedness: the edinburgh inventory," Neuropsychologia, vol. 9, no. 1, pp. 97-113, 1971. 
[57] A. Rey, "L'examen psychologique dans le cas d'encephalopathie traumatique," Archives de Psychologie, vol. 28, pp. 286-340, 1941.

[58] A. Rey, Manuel: Test de Copie d'une Figure Complexe, Centre de Psychologie Appliquee, Paris, France, 1959.

[59] P. Osterrieth, "Le teste de copie d'une figure complexe: contribution a l'etude de la perception et de la mémoire," Archives de Psychologie, vol. 30, pp. 206-256, 1944.

[60] A. Karapetsas and A. Kantas, "Visuomotor organization in the child: a neuropsychological approach," Perceptual and Motor Skills, vol. 72, no. 1, pp. 211-217, 1991.

[61] D. P. Waber and J. M. Holmes, "Assessing children's copy productions of the Rey-Osterrieth Complex Figure," Journal of Clinical and Experimental Neuropsychology, vol. 7, no. 3, pp. 264-280, 1985.

[62] C. Brandys and B. Rourke, "Differential memory abilities in reading- and arithmetic-disabled children," in A Critical Analysis. Neuropsychological Validation of Learning Disability Subtypes, B. P. Rourke, Ed., pp. 15-44, The Guilford Press, New York, NY, USA, 1991.

[63] M. Bryden and R. Steenhuis, "The assessment of handedness in children," in Neuropsychological Foundations of Learning Disabilities, J. Obrzut and G. Hynd, Eds., Academic Press, New York, NY, USA, 1991.

[64] D. Raczkowski, J. W. Kalat, and R. Nebes, "Reliability and validity of some handedness questionnaire items," Neuropsychologia, vol. 12, no. 1, pp. 43-47, 1974.

[65] S. Coren, "Measurement of handedness via self-report: the relationship between brief and extended inventories," Perceptual and Motor Skills, vol. 76, no. 3, pp. 1035-1042, 1993.

[66] N. J. Dorthe, T. D. Blumenthal, D. R. Jason, and P. E. Lantz, "The use of next-of-kin in assessing handedness," Perceptual and Motor Skills, vol. 81, no. 1, pp. 203-208, 1995.

[67] E. McMeekan and W. Lishman, "Retest reliabilities and interrelationship of the annett hand preference questionnaire and the edinburgh handedness inventory," British Journal of Psychology, vol. 66, pp. 53-59, 1975.

[68] B. J. Ransil and S. C. Schachter, "Test-retest reliability of the edinburgh handedness inventory and global handedness preference measurements, and their correlation," Perceptual and Motor Skills, vol. 79, no. 3, pp. 1355-1372, 1994.

[69] S. M. Williams, "Factor analysis of the edinburgh handedness inventory," Cortex, vol. 22, no. 2, pp. 325-326, 1986.

[70] D. Raczkowski, J. W. Kalat, and R. Nebes, "Reliability and validity of some handedness questionnaire items," Neuropsychologia, vol. 12, no. 1, pp. 43-47, 1974.

[71] W. J. Triggs, R. Calvanio, M. Levine, R. K. Heaton, and K. M. Heilman, "Predicting hand preference with performance on motor tasks," Cortex, vol. 36, no. 5, pp. 679-689, 2000.

[72] M. Verdino and S. Dingman, "Two measures of laterality in handedness: the edinburgh handedness inventory and the purdue pegboard test of manual dexterity," Perceptual and Motor Skills, vol. 86, no. 2, pp. 476-478, 1998.

[73] R. Steenhuis and M. Bryden, "Hand preference and performance: right-handers, left-handers and left-handers who are not consistently left-handed," Journal of Clinical and Experimental Neuropsychology, vol. 11, p. 37, 1989.

[74] M. B. Casey, E. Winner, I. Hurwitz, and D. DaSilva, "Does processing style affect recall of the rey-osterrieth and taylor complex figures?" Journal of Clinical and Experimental Neuropsychology, vol. 13, no. 4, pp. 600-606, 1991.
[75] F. Robichon, K. Giraud, M. Berbon, and M. Habib, "Sexual dimorphism in anterior speech region: an MRI study of cortical asymmetry and callosal size," Brain and Cognition, vol. 40, no. 1, pp. 241-246, 1999.

[76] G. Dirnberger, "Handedness is modulated by sex and selfperception, laterality: asymmetries of body," Brain and Cognition, vol. 17, pp. 275-186, 2012.

[77] F. Vargha-Khadem, E. Isaacs, S. van der Werf, S. Robb, and J. Wilson, "Development of intelligence and memory in children with hemiplegic cerebral palsy. The deleterious consequences of early seizures," Brain, vol. 115, no. 1, pp. 315-329, 1992.

[78] M. Annett, "The right shift theory of a genetic balanced polymorphism for cerebral dominance and cognitive processing," Current Psychology and Cognition, vol. 14, pp. 427-480, 1995.

[79] C. Gabbard, S. Hart, and V. Gentry, "General motor proficiency and handedness in children," Journal of Genetic Psychology, vol. 156, pp. 411-416, 1995.

[80] J. M. Piro, "Handedness and intelligence: patterns of hand preference in gifted and nongifted children," Developmental Neuropsychology, vol. 14, no. 4, pp. 619-630, 1998.

[81] H. Mayringer and H. Wimmer, "No deficits at the point of hemispheric indecision," Neuropsychologia, vol. 40, no. 7, pp. 701-704, 2002.

[82] D. Nettle, "Hand laterality and cognitive ability: a multiple regression approach," Brain and Cognition, vol. 52, no. 3, pp. 390-398, 2003.

[83] T. Björk, O. Brus, W. Osika, and S. Montgomery, "Laterality, hand control and scholastic performance: a british birth cohort study," British Medical Journal Open, vol. 2, no. 2, article e000314, 2012.

[84] A. Rodriguez, M. Kaakinen, I. Moilanen et al., "Mixedhandedness is linked to mental health problems in children and adolescents," Pediatrics, vol. 125, no. 2, pp. e340-e348, 2010.

[85] R. A. Yeo, S. W. Gangestad, and W. F. Daniel, "Hand preference and developmental instability," Psychobiology, vol. 21, no. 2, pp. 161-168, 1993.

[86] N. Cherbuin, P. Sachdev, and K. Anstey, "Mixed handedness is associated with greater age-related decline in volumes of the hippocampus and amygdala: the PATH through life study," Brain and Behavior, vol. 1, pp. 125-134, 2011.

[87] E. Luders, N. Cherbuin, P. M. Thompson et al., "When more is less: associations between corpus callosum size and handedness lateralization," NeuroImage, vol. 52, no. 1, pp. 43-49, 2010.

[88] I. Sommer, N. Ramsey, R. Kahn, A. Aleman, and A. Bouma, "Handedness, language lateralisation and anatomical asymmetry in schizophrenia: meta-analysis," British Journal of Psychiatry, vol. 178, pp. 344-351, 2001. 

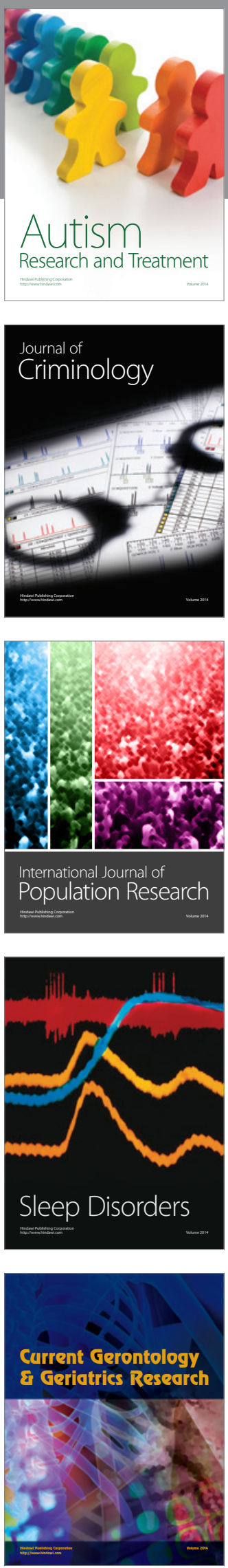
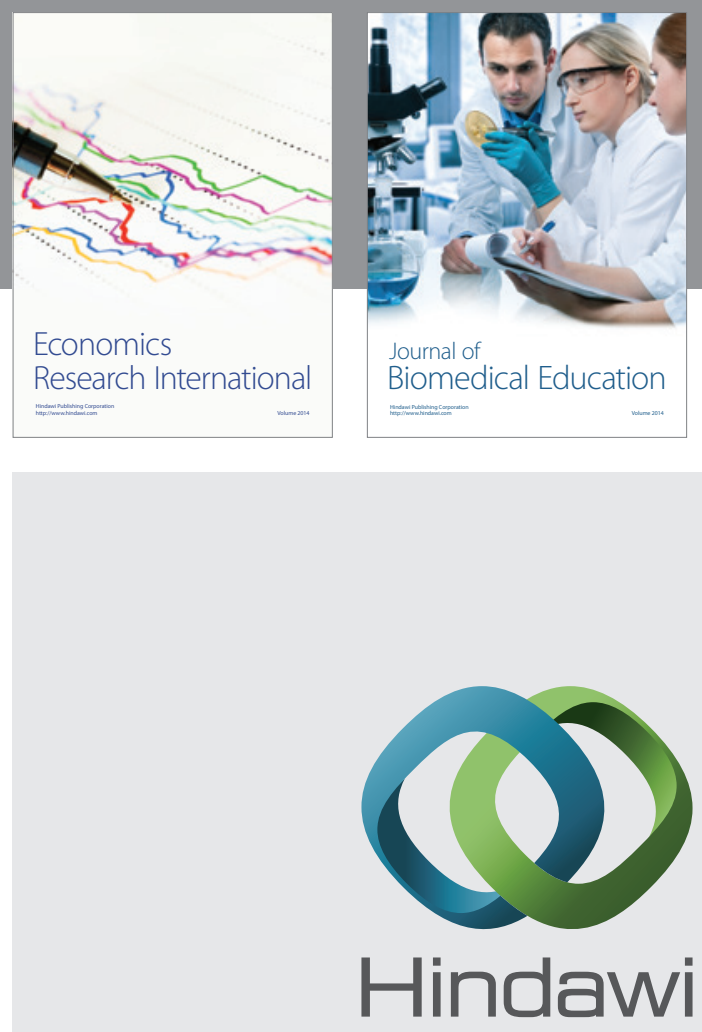

Submit your manuscripts at

http://www.hindawi.com
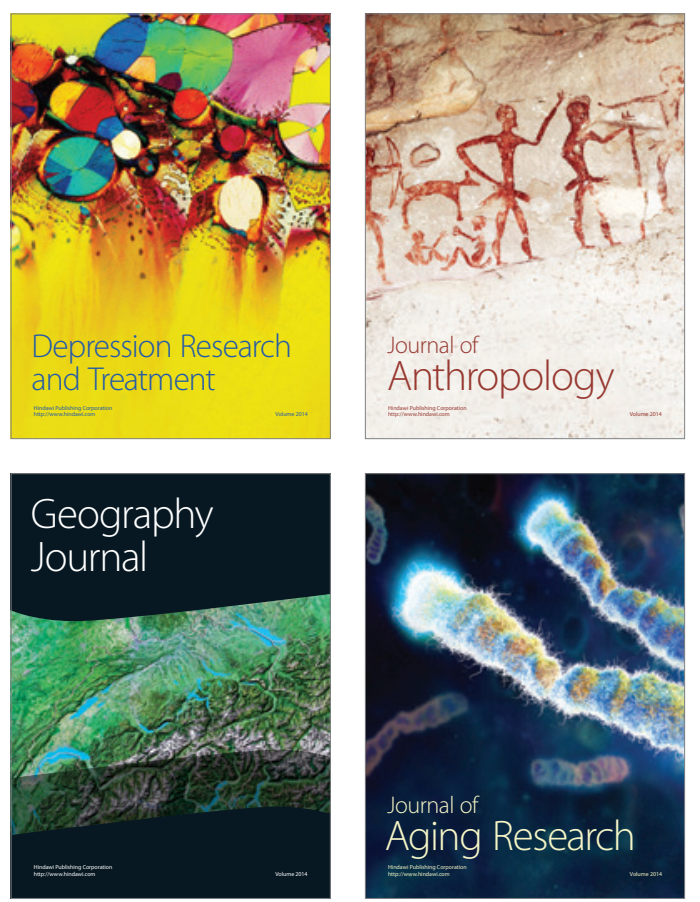
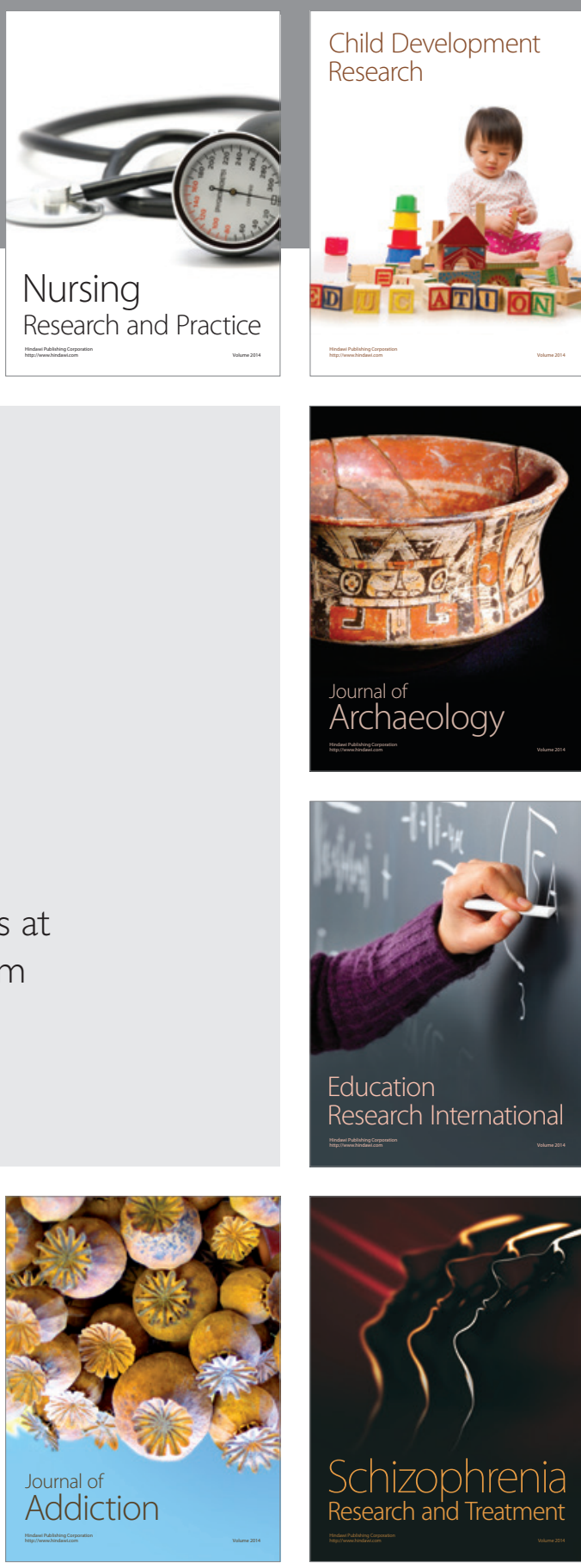

(D)
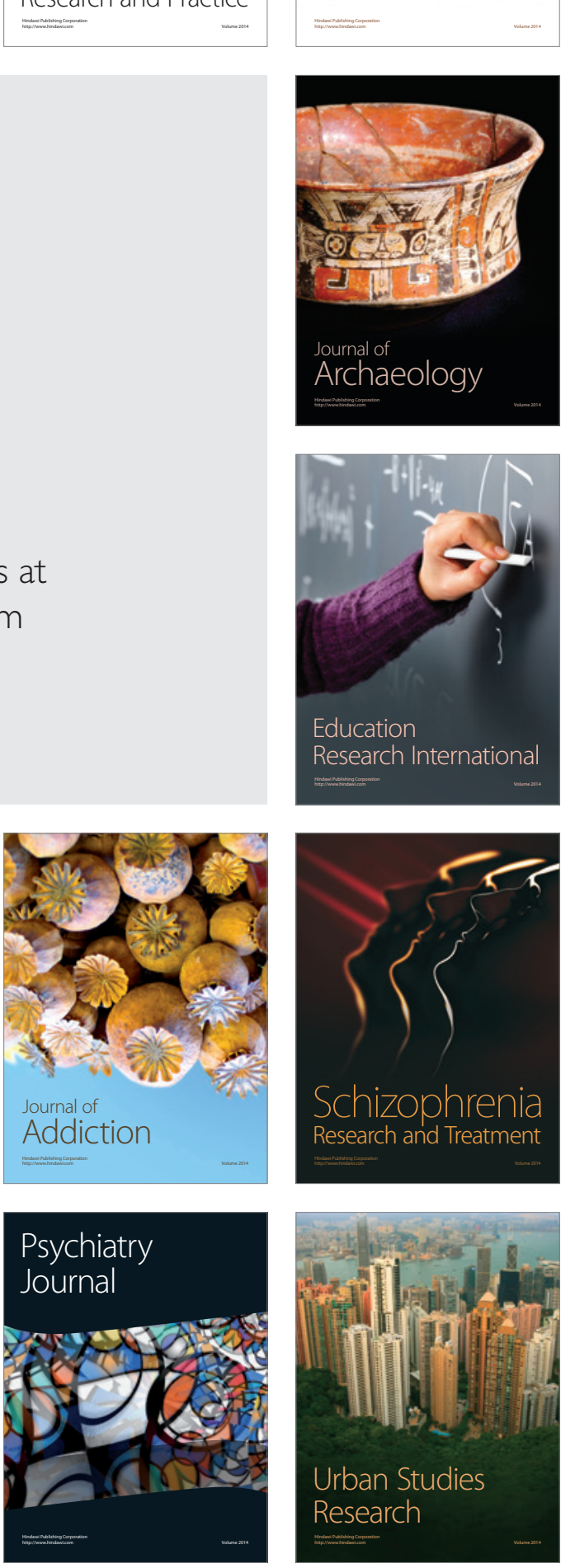